\title{
A New, Low-Energy Defibrillation Strategy: Use of Multiple Electric Field Directions to Reshape Scroll Wave Filaments
}

\author{
Kayleigh Wheeler ${ }^{1}$, Valentin Krinski ${ }^{2}$, Niels F Otani ${ }^{1}$ \\ ${ }^{1}$ Rochester Institute of Technology, Rochester, NY, USA \\ ${ }^{2}$ Max Planck Institute for Dynamics and Self-Organization, Gottingen, Germany
}

\begin{abstract}
There is continued interest in developing new, lowenergy methods for cardiac defibrillation. In this computational study, we consider the problem from a new perspective that, in contrast to several previous studies, leads to a strategy that works even when an arbitrarily large number of the rotating action potential waves are present during fibrillation, and works without requiring knowledge of the phases of these rotating waves. We show that sequences of one or more low-energy, electric field pulses, when designed with this strategy in mind, can convert the axes around which the waves rotate from a persistent form into forms that tend to shrink and disappear. When these sequences were applied with field strengths between 1.5 and $2.5 \mathrm{~V} / \mathrm{cm}, 1$ to 4 rotating waves representing fibrillation were terminated with success rates (76\%-90\%) much higher than those obtained using conventional stimuli (14\%). Limitations of this study include our use of a simplified model of cardiac geometry and our operation of the simulation in a positive filament tension regime that is also stable to electrical alternans. Nevertheless, we believe that the use of a sequence of electric fields designed to convert all persistent filament shapes to self-extinguishing ones is a promising idea that is worth further examination.
\end{abstract}

\section{Introduction}

Cardiac fibrillation, whether occurring in the atria or ventricles, is thought to be caused by several action potential waves that rotate or "reenter" the same areas of the tissue over and over, leading to a persistent, inefficient pattern of mechanical contraction that interferes with the normal pumping actions of the heart. Standard methods of defibrillation can be painful for the patient, damaging to tissue in and around the heart, and, in the case of an implantable device, draining on the battery. There is therefore current interest in designing low-energy forms of defibrillation. One approach receiving considerable attention places the electrodes, to the degree possible, in a configuration to create electric field pulses [1-3] rather than electrical stimuli delivered at one or more points on the tissue. Electric field pulses differ from point stimuli in that the goal is to create a more uniformly distributed electric field. This leads to a different response from the tissue. Notably, theory indicates that electric field pulses should launch new action potential waves from points and surfaces within the heart that represent heterogeneities in the gap junction conductances. These heterogeneities may therefore be thought of as "virtual electrodes," whose presence can be advantageous in the defibrillation process $[2,4,5]$. Such heterogeneities include the endocardial and epicardial surfaces, blood vessels within the heart muscle, plaques within the tissue, etc.

\section{Motivation and method}

Most theoretical work on the defibrillation-related properties of electric field pulses has focused on spatially twodimensional systems. In 2D, the interaction of the waves launched by the electric field with the rotating waves depends on the location of the rotating waves and where the waves are in their rotation when the electric field pulse is applied $[6,7]$. In contrast, in 3D, if a significant component of the electric field pulse is oriented along the axis of rotation of the rotating wave, then the result is, at some fundamental level, independent of where the wave is in its rotation $[8,9]$. The nature of this interaction is shown in Fig. 1. The electric field pulse causes an action potential plane wave to be launched from the rear surface. As it combines with the existing rotating wave, the axis of rotation (called a filament) is modified from an "I"-shaped configuration (meaning that the two ends of the axis attach to opposite surfaces, Fig. 1(a)) to one that is "L"-shaped (Fig. 1(b)) and later "C"-shaped (so that it is attached to adjacent surfaces-in this case, the front and top surfaces, Fig. 1(c)). Filaments behave like rubber bands in the socalled positive filament tension regime. Thus, L-, and Cshaped filaments, such as the one depicted in Fig. 1(c), as well as U- or O-shaped filaments, will tend to shrink and disappear along with the rotating waves they support, 


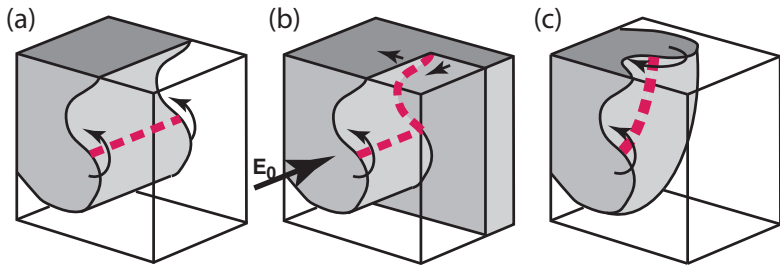

Figure 1. Illustration of the mechanism by which an electric field pulse, pointing parallel to an I-shaped filament, detaches the filament from a wall. (a) An action potential wave (in gray) rotates around an I-shaped filament prior to the application of the electric field. (b) Immediate effect of an electric field pulse pointed in the direction indicated by the arrow labeled $\mathbf{E}_{\mathbf{0}}$. (c) Effect of the electric field pulse on the rotating wave at a later time. The filament (i.e., axis of rotation) appears as a dashed pink line in all three panels.

while I-shaped filaments will tend to persist. Thus, the goal is to convert whatever I-shaped filaments are present during fibrillation into one of the other, more favorable shapes.

To create a situation in which only L-, C-, U- or Oshaped filaments are present, we have been developing electric field pulse protocols that depolarize the outside surface of the heart. The reason for this is shown in schematic form in Fig. 2. Assuming the heart is essentially a bowl-shaped system (the gray silhouetted object in Fig. 2), given any I-shaped filaments, as depicted by blue lines in Fig. 2(a), we would expect depolarization of the outside surface (shown in red in Fig. 2(b)) to detach the ends of the filaments connected to that surface, and redirect them to another surface, through the mechanism shown in Fig. 1. A little thought shows that these new filament shapes cannot be I-shaped, and so must be converted into one of the favorable shapes. Without other complications, these filaments should then shrink and disappear, terminating the fibrillation.

To investigate this idea, we constructed a simple com-

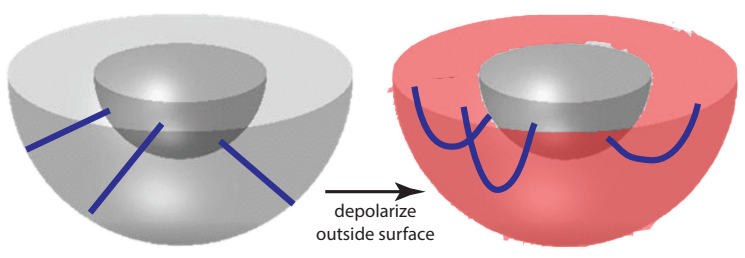

Figure 2. Expected change to I-shaped filaments when the outside surface of the heart is depolarized. (a) I-shaped filaments (blue lines) in a hemispherical shell system meant to model essential features of heart geometry. (b) Expected changes to the filaments (blue curves) when outside surface of the heart is depolarized (depicted in red). puter simulation model. Propagation of action potentials was simulated using the Barkley model [10], consisting of the equations,

$$
\begin{aligned}
& \frac{\partial u}{\partial t}=D \nabla^{2} u+\epsilon^{-1} u(1-u)(u-(v+b) / a) \\
& \frac{\partial v}{\partial t}=u-v
\end{aligned}
$$

Here $u$ and $v$ represent the membrane potential and the refractoriness of the tissue, respectively. The parameters are defined to be $a=0.8, b=0.05$ and $\epsilon=0.02$, which puts the system in the positive filament tension regime [11]. We used a grid spacing of 0.167 and a timestep of $1.6 \times 10^{-3}$. We chose the system shape to be a hemispherical shell of finite thickness, because it is simple to model, yet retains key geometric properties of the heart. This shape was created by defining the simulation system to be a 3-d rectangular grid, and then defining the gap-junction conductivity $D$ to be 1.0 between adjacent nodes when both lay within the hemispherical shell region, and 0.0 otherwise.

The system was initiated with 1 to 4 rotating action potential waves, initiated at random locations with random phases, meant to represent fibrillation. One or more electric field pulses, pointing in different directions, were then applied to the system. The effect of electric field pulses was modeled by modifying the boundary conditions [4] on the hemispherical shell, defining them to be $\hat{\mathbf{n}} \cdot \nabla u=\hat{\mathbf{n}} \cdot \mathbf{E}_{0}$ during a pulse with electric field $\mathbf{E}_{\mathbf{0}}$, and $\hat{\mathbf{n}} \cdot \nabla u=0$ otherwise. Here, $\hat{\mathbf{n}}$ represents an outward pointing normal unit vector. Since we are focusing on weak electric fields in this study, we assumed that only the system surfaces are affected by the electric field pulses, and not smaller heterogeneities.

\section{Results}

To calibrate our system, we first tried electric field pulses directed perpendicular to the axis of symmetry, in the $\hat{\mathbf{y}}$ direction. (Figure 4 shows coordinate axis directions.) This field direction is roughly analogous to the one one would obtain if the heart were shocked using an external defibrillator with paddles placed to the left and right of the heart. (Of course, external defibrillators employ much higher field strengths than we are using here.) The field pulse was applied with 5 different timings relative to the waves' rotation periods, with 1 to 4 rotating waves initially present. As shown in the column of the table in Fig. 3 devoted to the $y$-directed field, when low energies are used, failure to defibrillate is nearly complete: Of the 80 trials we conducted with field strengths between 1.54 and 2.46 $\mathrm{V} / \mathrm{cm}$, only 11 (14\%) of the different combinations of stimulus timing and number of waves could be successfully terminated. In contrast, when a single radially directed electric field pulse was used (as shown by the red arrows in 

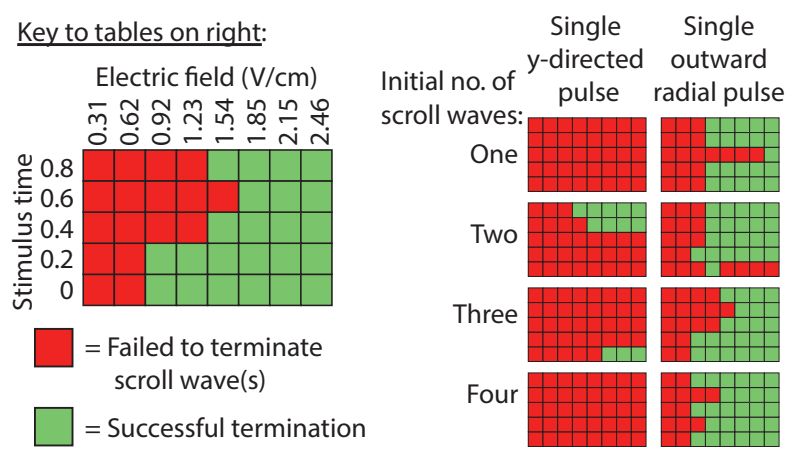

Figure 3. Summary of results for the cases of a single electric field pulse in the $y$-direction (first column) and a single radial field pulse (second column). Each pulse was applied with different field strengths ranging from 0.31 to $2.46 \mathrm{~V} / \mathrm{cm}$ at five different times, equally spaced within a rotating wave period. Red and green denote failed and successful defibrillation (i.e., rotating wave termination), respectively. Stimulus times are in units of the rotating wave period.

Fig. 4(c) and (d)), the success rate was much higher, with all waves terminated in 72 of the 80 trials $(90 \%)$ having field strengths between 1.54 and $2.46 \mathrm{~V} / \mathrm{cm}$, as shown in the right column of Fig. 3.

We also found that the radial field pulse terminated the rotating waves in the manner expected. Figure 4(a) shows the initial presence of three rotating action potential waves, representing fibrillation. The I-shaped filaments around which these waves were rotating are shown in Fig. 4(b). Figure 4(c), shows the immediate effects of the radial electric field pulse. The entire outside surface of the system is seen to be depolarized. Simultaneously, we see in Fig. 4(d) that the three I-shaped filaments have been detached from the outside wall, and have reconfigured themselves into two U-shaped filaments. These filaments were observed to shrink and disappear. The accompanying waves terminated shortly thereafter.

Other electric field pulse sequences were also tried. When a single, $(-z)$-directed pulse was tried, nearly all, but notably, not all of the entire outside surface, was depolarized. Because $\hat{\mathbf{n}} \cdot \mathbf{E}_{0} \approx 0$ just under the rim of the hemispherical shell, filaments reattached themselves to this region of the outside surface, and therefore remained Ishaped. The success rate was therefore low: $(15 / 80,19 \%)$. We also tried two series of five pulses. When the five pulses were delivered in rapid succession in the following directions: $-z,+x,-x,+y$ and $-y$, separated by quiescent intervals of $3.4 \mathrm{~ms}$, we obtained a success rate of $76 \%$ $(61 / 80)$. Similarly, when the pulses were delivered in the sequence $-z,+x,+y,-x$ and $-y$, with no quiescent intervals between pulses, we obtained a similar success rate $(81 \%, 65 / 80)$. While these two sequences were again de-
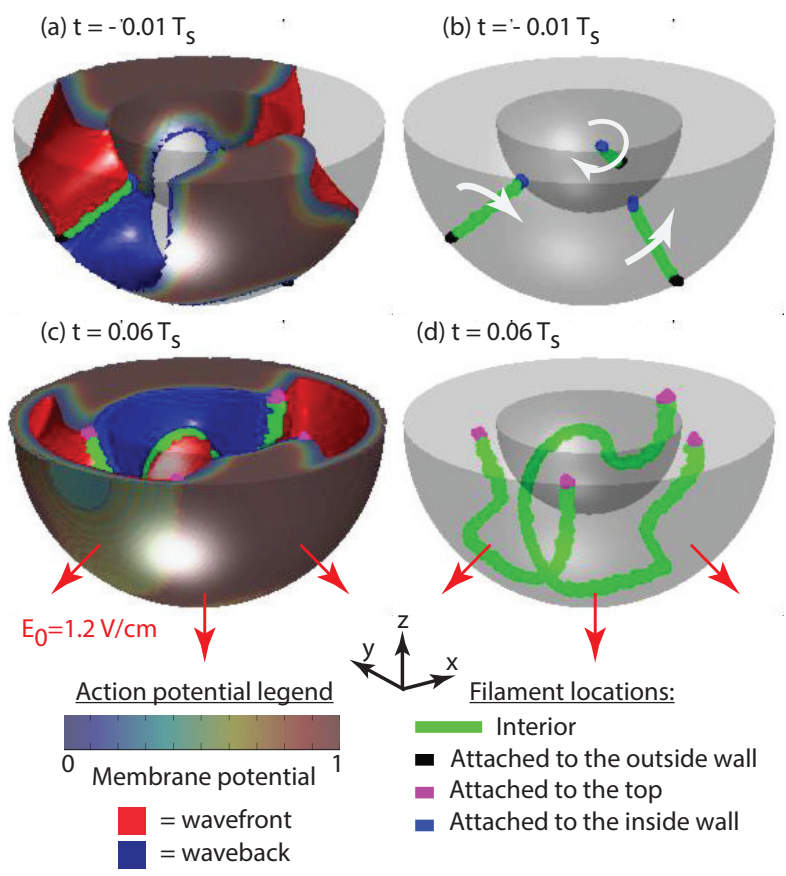

Figure 4. (a) Action potentials and (b) filaments of three rotating waves just before the delivery of the stimulus. (c) Action potentials and (d) filaments during the radial electric field stimulus pulse.In the action potential plots, the region occupied by the action potentials is opaque, with wavefronts and wavebacks colors bright red and bright blue, respectively, and intersection of the action potentials with the boundaries colored with muted colors representing the value of the membrane potential. The location of the filaments are shown in green in all four plots, but are best seen in panels (b) and (d). Intersection of the filaments with the surfaces are colored with different colors, as indicated by the legend. White arrows in panel (b) show the sense of rotation around the corresponding filaments. Red arrows in panels (c) and (d)

signed to effectively depolarize the entire outside surface, whether this effect was completely achieved was unclear, since the regions of depolarization, once established by each pulse, propagated inward from the outside surface, leading to a complicated pattern of depolarization. These multiple pulse methods were tried, because they might be easier in practice to implement than the radial field pulse.

These methods were not always successful, apparently for two major reasons. First, it is possible for a filament, in the process of shrinking, to find itself too close to a system boundary. It can then reattach to this boundary, in some cases, splitting the filament in two, and in some cases, changing one or both of the filaments' configurations back to I-shaped. Second, it is possible for two filaments to reconnect with each other, making it possible for 
both filaments to change their shapes. We observed that the reconnection process can either create or eliminate Ishaped filaments.

\section{Conclusions}

We have found that depolarizing the entire outside surface of a simple geometric model of the heart is able to terminate one to four rotating waves representing fibrillation. The method works by a three-step process: (1) one or more electric field pulses are used to depolarize the entire outside surface, detaching all filaments from that surface, which then (2) converts persistent I-shaped filaments into C-, U-, or O-shaped filaments, which are known to (3) shrink and disappear in the positive filament tension regime. We applied a number of different electric field stimulus pulse protocols in order to test this method. The protocols that were the most effective were those that were designed to depolarize the entire outside surface. Furthermore, termination of the wave was observed to occur by the mechanism predicted, through the three-step process outlined above. The method was not always successful, because filaments sometimes reattached to nearby surfaces, recreating I-shaped filaments, and sometimes reconnected with each other to form I-shaped filaments. However, in cases when the sequence of electric field pulses were successful in initially detaching filaments from the outside wall, the success rates were high- $76 \%$ to $90 \%$. Field strengths required to detach filaments from the outside wall, leading to successful terminations, were quite low: 1.5 to $2.5 \mathrm{~V} / \mathrm{cm}$. Notably, unlike many two-dimensional studies of the electric field pulse defibrillation, our method requires no knowledge of the number of rotating waves present, and no knowledge of their locations or phases when the pulse or pulses are applied.

This study has a number of limitations. The system was assumed to be in the positive filament tension regime, which may or may not be the case when the heart is in fibrillation. Our simulations employed a simple, hemispherical shell model of the heart, which likely does not include all geometric features that could be important in the defibrillation process. We have not tested the cases in which wave instabilities such as action potential duration alternans or wave breakup are present. Finally, our model does not include cardiac fiber rotation, an inherent property of cardiac tissue, which is known to modify the properties of filaments. In view of these limitations, we do not claim at this stage that this is a valid defibrillation method. Rather, we put forth this method as a new idea, which we hope will advance the study of low-energy defibrillation.

\section{References}

[1] Ripplinger CM, Krinsky VI, Nikolski VP, Efimov IR. Mechanisms of unpinning and termination of ventricular tachycardia. American Journal of Physiology Heart and Circulatory Physiology 2006;291:H184-H192.

[2] Fenton FH, Luther S, Cherry EM, Otani NF, Krinsky V, Pumir A, Bodenschatz E, Gilmour Jr. RF. Termination of atrial fibrillation using pulsed low-energy far-field stimulation. Circulation 2009;120:467-476.

[3] Luther S, Fenton FH, Kornreich BG, Squires A, Bittihn P, Hornung D, Zabel M, Flanders J, Gladuli A, Campoy L, Cherry EM, Luther G, Hasenfuss G, Krinsky VI, Pumir A, Gilmour Jr. RF, Bodenschatz E. Low-energy control of electrical turbulence in the heart. Nature 2011;475:235239.

[4] Pumir A, Krinsky V. Unpinning of a rotating wave in cardiac muscle by an electric field. J Theor Biol 1999; 199:311-319.

[5] Pumir A, Nikolski V, Horning M, Isomura A, Agladze K, Yoshikawa K, Gilmour R, Bodenschatz E, , Krinsky V. Wave emission from heterogeneities opens a way to controlling chaos in the heart. Phys Rev Lett 2007;99:208101.

[6] Otani NF. Termination of reentrant cardiac action potential ropagation using far-field electrical pacing. IEEE Transactions on Biomedical Engineering 2011;58(7):2013-2022.

[7] Hornung D, Biktashev VN, Otani NF, Shajahan TK, Baig T, Berg S, Han S, Krinsky VI, Luther S. Mechanisms of vortices termination in the cardiac muscle. Royal Society Open Science 2017;4:170024.

[8] Biktashev VN. Evolution of vortices in active media. Ph.D. thesis, Moscow Institute of Physics and Technology, 1989.

[9] Zemlin C, Mironov S, Pertsov A. Delayed success in termination of three-dimensional reentry. Journal of Cardiovascular Electrophysiology 2003;14(10, Supplement):S257S263.

[10] Barkley D. Linear stability analysis of rotating spiral waves in excitable media. Phys Rev Lett 1992;68(13):2090-2093.

[11] Alonso S, Sagues F, Mikhailov AS. Taming Winfree turbulence of scroll waves in excitable media. Science 2003; 299:1722-1725.

Address for correspondence:

Niels F. Otani

Rochester Institute of Technology

School of Mathematical Sciences

Lomb Memorial Drive

Rochester, NY 14624, U.S.A.

nfosma@rit.edu 